\section{POLYTENE CHROMOSOMES}

\section{Selective Replication}

from our Molecular Genetics Correspondent

FORMATION of the giant polytene chromosomes of the salivary glands of Drosophila takes place by several successive replications of the diploid genetic material. By the time the final number of nine replications has been achieved, each polytene chromosome has more than one thousand times its usual DNA content, for each successive replication doubles its amount of DNA. But some sequences suffer more doublings than others; a comparison of the amount of DNA in the euchromatic arms of the polytene chromosomes with the DNA content of the chromocentre which is formed by the aggregation of regions of heterochromatin shows that the more condensed heterochromatic DNA does not keep pace with the euchromatic sequences; instead of eight to nine doublings it is replicated at most only a very small number of times.

Prompted by this split in the control of replication in salivary gland cells, Spear and Gall (Proc. natn. Acad. Sci., U.S.A., 70, 1359; 1973) have examined the replication of the sequences which code for ribosomal RNA. The two hundred or so genes which specify ribosomal RNA are clustered on the sex chromosomes at or close to the site where the nucleolar organizer forms to synthesize ribosomal RNA. This region, although itself replicated during the formation of polytene chromosomes (as shown by incorporation of ${ }^{3} \mathrm{H}$-thymidine) and active in the transcription of ribosomal RNA molecules, is surrounded by the heterochromatin which is replicated to a lesser extent and is not transcribed at all.

Control of replication in polytene Drosophila cells identifies three types of sequence. In a previous article, Gall, Cohen and Polan (Chromosoma, 33, 319 ; 1971) used hybridization with satellite DNA to show that these sequences-a major part of the heterochromatin-appear to replicate hardly at all compared with the extensive polytenization of sequences contained within heterochromatin. Spear and Gall now show that ribosomal RNA genes fall into neither of these categories, but are controlled independently.

Cells of Drosophila which have the $\mathrm{XO}$ constitution (that is an $\mathrm{X}$ chromosome and no $\mathrm{Y}$ chromosome) have only one nucleolar organizer, whereas female cells of the XX class have two nucleolar organizers. By measuring the extent of hybridization with ${ }^{3} \mathrm{H}-\mathrm{rRNA}$ of DNA extracted from these two types of diploid cell, Spear and Gall found that the content of rDNA is proportional to the number of nucleolar organizers. In one strain of $D$. melanogaster they found that XO cells have $0.26 \%$ and $\mathrm{XX}$ cells have $0.47 \% \mathrm{rDNA}$; in another strain the XO cells have $0.24 \%$ and the XX cells have $0.37 \%$ rDNA. This shows that a nucleolar organizer forms at each cluster of rRNA genes on an X chromosome, the two clusters in $\mathrm{XX}$ cells presumably being identical.

But very different results were obtained when the DNA used for hybridization was extracted from polytene cells of the salivary gland. In both strains of D. melanogaster, only $0.08 \%$ of the DNi corresponded to ribosomal RNA. In a polytene cell with the 1,024 eqivalents of euchromatic DNA produced by nine doublings of the diploid content, this proportion corresponds to about 150-200 times the amount of ribosomal DNA in a diploid cell. This would be generated by some six or seven doublings of the genes coding for ribosomal RNA.

But the control of replication of the ribosomal RNA genes is not merely set at a lower level than that for euchromatic DNA. In both $\mathrm{XO}$ and $\mathrm{XX}$ cells, the rDNA content of polytene nuclei corresponded to the same level of $0.08 \%$. The ribosomal RNA genes have therefore doubled one more time in the XO cells to compensate for their lower starting value; the final level of rDNA in these cells is therefore independent of the number of nucleolar organizers with which their diploid ancestors started. In contrast to this behaviour, the remaining sequences of the $\mathrm{X}$ chromosome double only the same fixed number of times in XO cells and XX cells.

Ribosomal genes of these cells are therefore not only under a control independent from that of euchromatin and heterochromatin but also achieve a fixed final level (relative to the number of doublings of euchromatin) instead of doubling a certain number of times. That the control of ribosomal RNA genes is especially important in the cell has been shown also by the phenomenon of magnification discovered by Ritossa, in which mutants with a reduced number of ribosomal RNA genes seem to be able to increase the number in germ line cells so that the wild type situation is restored. Both lateral extension of the number of genes in one chromosome and the multiplication of the number of chromosome IDNA regions are therefore utilized to maintain a specific content of ribosomal RNA genes in different circumstances.

\section{BACTERIOLOGY \\ Hospital Bugging}

from a Correspondent

HeXachlorophane has been under attack because it may lead to neurological disorders if used in large amounts for washing babies, and it has been withdrawn from most germicidal soaps. But hospital staff still find it valuable for hand washing and preoperative

\title{
Haemoglobin Prediction Verified
}

The survey by Perutz of abnormal human haemoglobins has revealed that whenever the mutation involves only an amino acid on the outside of the molecule there are no aberrations in the functional characteristics. An exception to this rule was the $\beta$-chain variant, haemoglobin Seattle, which was reported to have glutamic acid in place of alanine at position 76. The side chain in this position is on the surface, and not apparently involved in any intramolecular interactions. Thus either Perutz's scheme of the structure and function relation contained a flaw, or the fingerprinting analysis as published was in error.

The second explanation has now been proved correct. Kurachi et al., writing in Nature New Biology next Wednesday (June 27), report the results of a reexamination of the deviant peptide component in the fingerprint map. They find that rather than a glutamic acid for an alanine at position 76, haemoglobin Seattle has aspartic acid for alanine at position 70, which in Perutz's model is in contact with the haem group. The functional disturbance which this substitution induces is not large, the haem- haem instructions and Bohr effect being normal. There is, however, a decrease in oxygen affinity, and an enhanced tendency towards oxidation to methaemoglobin.

In an accompanying letter, Anderson, Perutz and Stamatoyannopoulos describe the structural consequences of the replacement. The difference electron density map reflects the substitution itself, and in addition a displacement of another aspartate side chain and a small disturbance of the haem group, which is tentatively identified in terms of an alteration in the tilt. In the deoxy-form the $\beta$ carbon and the carboxyl group of the anomalous aspartate side chain are close to two different methyl side chains of the haem. One of these contacts would be lost in the transition of the conformation to that of the oxygenated form.

It is not obvious why these distortions would have the effect that they do on the properties. Anderson et al. conjecture that the negative carboxylate group may polarize the haem sufficiently to affect the oxygen affinity, and the increased negative charge could also make it more susceptible to oxidation. 